\title{
Switchable window based on electrochromic polymers
}

\author{
Chunye $\mathrm{Xu},{ }^{\text {a) }}$ Lu Liu, Susan E. Legenski, Dai Ning, and Minoru Taya \\ University of Washington, Seattle, Washington 98195
}

(Received 3 November 2003; accepted 17 March 2004)

\begin{abstract}
A large contrast ratio $(>\Delta \mathrm{T}=60 \%)$ and rapid switching $(0.3-1 \mathrm{~s})$ electrochromic (EC) polymer device that consists of a laminated two-layer structure between two electrodes was prepared. The new design consists of an indium tin oxide (ITO) glass electrode, a cathodic EC polymer film, a solid electrolyte, and a counterelectrode that replaces the anodic EC polymer and ITO electrode. Four EC polymers including two new EC polymers, Poly[3-methyl-3'-propyl-3,4-dihydro-2H-thieno(3,4-b)(1,4)dioxepine] (PProDOT-MePro) and Poly[3,3-diethyl-3,4-dihydro-2H,7H-(1,4)dioxepino(2,3-c)pyrrole] (PProDOP-Et ${ }_{2}$ ), were synthesized as cathodic EC polymers. A carbon-based counterelectrode was prepared for comparison with an Au-based counterelectrode. Several kinds of polymer gel electrolytes were prepared for comparison. The devices (windows) were increased in area from $0.028 \times 0.04 \mathrm{in.}^{2}, 1 \times 1 \mathrm{in}^{2}$ to $3 \times 3 \mathrm{in.}^{2}$ Three main components, the EC polymer film, the gel electrolyte, and the counterelectrode, were studied and their optical properties, conductivities, and repeatabilities were compared. The effects of window size on the contrast ratio, switching speed, power usage, and repeatability were studied.
\end{abstract}

\section{INTRODUCTION}

Switching technology is a continuously growing field due to its current and potential applications for architectural, vehicular, and aircraft windows, skylights, sunroofs, eyeglasses, and numerous types of displays. ${ }^{1,2}$ Whether its purpose is for energy conservation, functional use, or purely aesthetic, switchable devices have a promising future. Generally, these devices can be categorized into three main groups based on the active material present: suspended particles, liquid crystals, and electrochromics. ${ }^{3}$ On a macroscopic structure level, the device design is the same for all three groups: an active material (liquid crystal medium, suspended particle or electrochromic material) is sandwiched between two electrodes. When a potential is applied, the optical properties of the devices change. However, on a microscopic level, the switching mechanisms are quite different. To use switching devices for practical applications, it is imperative that the devices demonstrate large contrast ratios, rapid switching, repeatability, and an overall robust design. Here, a comparison between three main switching devices is presented regarding their resulting performance.

One recent design has surfaced to minimize some of of the constraints of liquid crystal displays (LCDs). Polymer-dispersed liquid crystal (PDLC) devices include

\footnotetext{
a) Address all correspondence to this author.

e-mail: chunye@u.washington.edu

DOI: 10.1557/JMR.2004.0259
}

an active layer composed of liquid crystal droplets encapsulated within a transparent matrix. The on-off switching states yield a translucent white state and a transparent state, respectively. The transparency of the device is greatly improved because no polarizer is needed. Also, manufacturing constraints are eased because the exact thickness of the film is not important. ${ }^{3}$ However, the PDLC design, like traditional LCD designs, still cannot be viewed at an angle, requires high voltage, and is costly in production. The change in transmittance $(\Delta \mathrm{T})$ ranges from $50-80 \%$, but more recent work is geared toward increasing opacity by adding dyes. $^{3}$

Suspended particle devices encompass an innovative approach to switchable devices. Originally patented in 1934 by Dr. Edwin Land, the active layer in the suspended particle device (SPD) consists of needle-shaped dipole particles suspended in a polymer matrix. SPDs are viewed as an improvement from LCDs because they require no polarizing sheets, are specular, and ease production constraints due to their simplified assembly. Advantages of SPDs include its fast switching times (100-200 ms), its high transmission ranges, and capacity to achieve intermediate transmissive states by varying the voltage. However, the large disadvantage is the high voltage needed to operate the devices (35-100 VAC). SPDs also have no memory effect; without an electric field, the device will return and remain in the off-state until the electric field is resumed. ${ }^{4}$ Another disadvantage is its limited color. 
Electrochromics are materials that change their color when a potential is applied due to an electrochemical reaction. ${ }^{5}$ Electrochromic materials can be divided into many different classes. Some of the more heavily researched classes include both the inorganic and organic systems, such as transition metal oxides and polynuclear transition metal hexacyanometallates, and the organic systems, viologens and conducting polymers. But altogether, electrochromics are the most popular switching technology for large-area switching devices.

Tungsten trioxide film $\left(\mathrm{WO}_{3}\right)$ is one example of a transition metal oxide. Electrochromism was first reported in $\mathrm{WO}_{3}$ films in 1969. At best, the switching time is of the order tens of seconds for even small-area devices. Also, the performance of transition metal oxide electrochromic devices is $\mathrm{pH}$-dependent, moisturedependent, and sensitive to exposure to the atmosphere. ${ }^{6}$ This can lead to difficulties in processing this inorganic electrochromic devices (ECD).

Conducting polymers have been the most promising of organic electrochromics due to the ease in processing, unlike their inorganic counterparts. Also, there is a high degree of color tailorability by fine tuning of the energy gap and overall much higher coloration efficiencies. Polymer-based ECDs prove more advantageous than previously described devices for several reasons: they require power only during switching; small switching voltages (1-5 V) are adequate for operation; always specular; low polarization; long optical memory; and ease in processing/fabrication over large areas.

The conventional design of an electrochromic (EC) polymer window system is a three-layer structure that consists of cathodic EC polymer, for example, poly $(3,4-$ ethylenedioxythiophene) (PEDOT) or poly(3,4 propylenedioxythiophene) (PProDOT) and their derivatives, an anodic EC polymer, for example, PBEDOT-NMeCz and a solid electrolyte between two electrodes (Fig. 1). ${ }^{6,7}$ This EC device consists of dual polymers, and while the opacity of the colored state is increased, the transmittance of the bleached state is decreased. In addition, the anodic EC monomer (e.g., BEDOT-NMeCz) is difficult to synthesize and its transparent state yields a yellow color. On

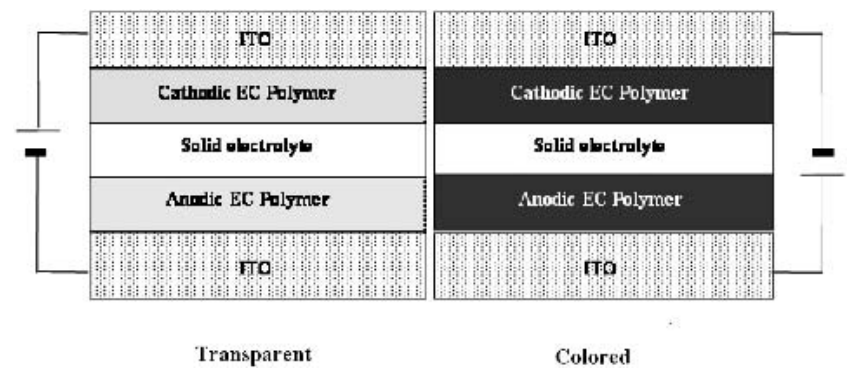

FIG. 1. The conventional design of a color changeable EC polymer device including three layers: a cathodic EC polymer film, an anodic EC polymer film, and a solid electrolyte layer between two electrodes. the other hand, poly (3,4-propylenedioxythiophene) derivative, PProDOT- $\mathrm{Me}_{2}$, shows a $\Delta \% \mathrm{~T}$ of close to $78 \%$, which is significantly higher than the $62 \%$ of PProDOT or the $45 \%$ of PEDOT. To the best of our knowledge, this value constitutes the highest contrast reported in the literature to date for an EC polymer. Furthermore, it exhibits rapid switching, low oxidation potentials, and greater stability at ambient and modest elevated temperatures. ${ }^{7}$ Here we propose a two-layer structure design that consists of only one EC polymer film and a solid electrolyte between two electrodes; an Au-based (or carbon-based) counterelectrode replaces the anodic EC polymer and indium tin oxide (ITO) electrode. Together, they can improve the color change speed and the contrast ratio of visible light transmittance between the two states.

\section{EXPERIMENTAL}

\section{A. Synthesis of cathodic EC monomer}

The synthesis of low oxidation potential monomers, which can undergo facile electropolymerization to yield conducting and electroactive EC polymers, is an initial step of this research.

\section{Cathodic blue color monomer}

a. Cathodic monomer, ProDOT-Me $\mathrm{Me}_{2}$ (Blue 1)

The reaction proceeds well for cathodic monomer, ProDOT- $-\mathrm{Me}_{2}$; the substitution would have to proceed on a neopentyl-type structure, the processing of which is known to be difficult. ProDOT- $\mathrm{Me}_{2}$ was successfully synthesized using the transetherification reaction. ${ }^{2,7}$ The monomer was characterized by ${ }^{1} \mathrm{H}$ NMR spectroscopy $\left(\mathrm{CDCl}_{3}\right)$ as $\delta 1.02(\mathrm{~s}, 6 \mathrm{H}), 3.73(\mathrm{~s}, 4 \mathrm{H}), 6.48(\mathrm{~s}, 2 \mathrm{H}){ }^{2}$

\section{b. Cathodic monomer, ProDOT-MePro (Blue 2)}

The following is the synthetic route. The starting materials were 3,4-dimethoxylthiophene. Double Williamson etherification reaction was used. The product was obtained in good yields. This double Williamson etherification has been used for such purpose by others to make 3,4-dialkyldioxylthiophene derivatives. The derivatives reported in literature all have symmetric structure. The new compound we have obtained (Fig. 2) is not only one new compound but also has asymmetric structures, which is also new.
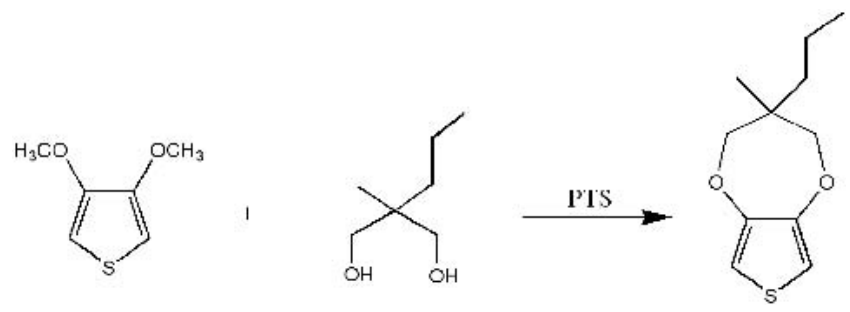

FIG. 2. The synthetic route of the new thiophene based EC monomer. 


\section{Cathodic red color monomer}

a. Cathodic monomer, ProDOP- $\left(\mathrm{CH}_{3}\right)_{2}$ (Red 1)

One cathodic monomer, ProDOP- $\left(\mathrm{CH}_{3}\right)_{2}$, was synthesized according to the procedures in literature ${ }^{9-11}$ with some modifications. ${ }^{1,2}$

\section{b. Cathodic monomer, ProDOP-Et ${ }_{2}($ Red 2)}

Figure 3 shows the synthetic route of the new pyrrolebased EC monomer, which is Poly[3,3-diethyl-3,4dihydro-2H,7H-(1,4)dioxepino(2,3-c)pyrrole] (PProDOP-Et $)_{2}$. The starting material was made in our lab according to the literature. ${ }^{12}$ The yield of each step shown in Fig. 3 is good (54 92\%). A similar route has been used for synthesis of this type of monomer. Usually, dibromides and disulfonate were used in the first step, which offered low yields. The first step was modified by using double Mitsunobu reaction and much better yield was obtained. The resulting monomer is a new one.

The monomer can be electrochemically polymerized in nonaqueous electrolyte solution. The resulting film shows a pinkish red color. The film can be switched between color and transparent states repeatedly, resulting in high contrast ratio.

\section{B. Gel electrolyte preparation}

The electrolytes must be ionically conductive, but electronically insulating. ${ }^{13-16}$ For a gel electrolyte to be suitable for smart window applications, a high ionic conductivity and transparency are two key requirements.

Poly(methyl metracrylate) (PMMA)-based gel electrolytes were prepared and differed by the salt and solvents used. The solid polymer matrix of PMMA provides dimensional stability to the electrolyte while the high permittivity of the solvents enables extensive dissociation of the salts. The salts that were investigated include lithium perchlorate $\left(\mathrm{LiClO}_{4}\right)$, tetrabutyl ammonium perchlorate (TBAP), and trifluorosulfonimide $\left[\mathrm{LiN}\left(\mathrm{CF}_{3} \mathrm{SO}_{2}\right]_{2}\right.$. The solvents used were propylene carbonate (PC), ethylene
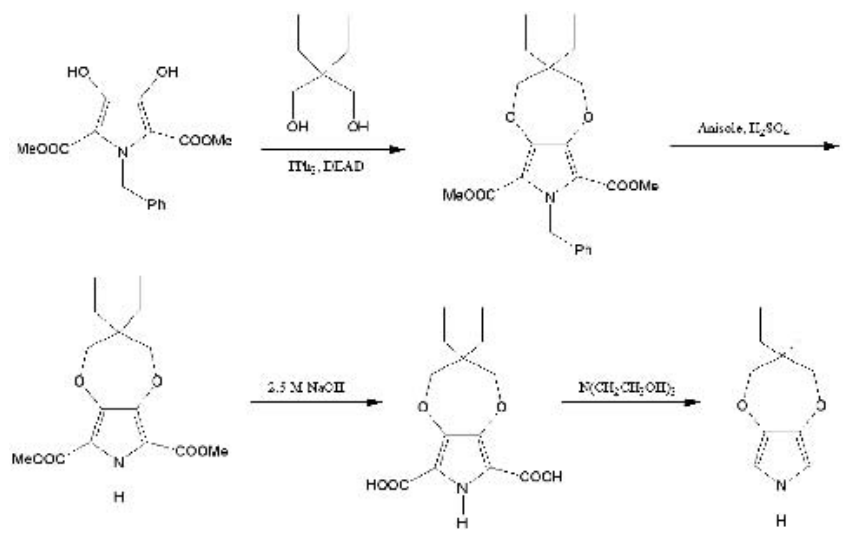

FIG. 3. The synthetic route of pyrrole based new EC monomer. carbonate (EC), acetonitrile, and $\gamma$-butylactone (GBL) and were significantly dried over molecular sieves before use. The gel electrolytes were synthesized by first dissolving the salt in the solvent and then adding the PMMA. A highly conductive $(2 \mathrm{mS} / \mathrm{cm})$, viscous and transparent $(88 \%)$ gel electrolyte was formed.

\section{Design of counterelectrode}

\section{Au-based counterelectrode}

Polished float glass (Delta Technologies Limited, Stillwater, MN, Limited, $0.7-\mathrm{mm}$ thick) was used as a substrate for the Au-based counterelectrode. The glass was cut into 4-inch-diameter wafers for photoresist spin coating. Lithography and sputtering were used to form the Au pattern on the glass wafer. Before the Au sputtering, TiW was first sputtered on the glass substrate to tightly bind Au to the glass substrate.

\section{Carbon-based counterelectrode}

The carbon-based counterelectrode was prepared for comparison with the Au-based counterelectrode. The substrate for the carbon coating is a polished float glass cuvette plate coated with ITO on one side with $\mathrm{R}_{\mathrm{s}}<10 \Omega$ (Delta Technologies, Limited, 0.7-mm thick). The carbon material used is HITASOL GA.66M [Hitachi Powdered Metals Co. Ltd. (HPM), Chiba, Japan]. Its electrical conductivity is not less than $10^{-2} \mathrm{~S} / \mathrm{cm}$. The covering percentage of the carbon materials is below $20 \%$ to maintain high visible light transmittance.

\section{Device assembly}

First, slides of $\mathrm{L} \times \mathrm{W} \times \mathrm{T}=50 \times 7 \times 0.7 \mathrm{~mm}^{3}$, $25.4 \times 25.4 \times 0.7 \mathrm{~mm}^{3}$, and $75 \times 75 \times 0.7 \mathrm{~mm}^{3}$ were cut from an Au-patterned glass wafer and a carbon-patterned ITO glass plate. The gel electrolyte was uniformly placed between the cathodic EC polymer deposited ITO slide and the counterelectrode to form the device. A special rubber was used for tightly sealing the device.

\section{MECHANISM FOR COLOR CHANGE OF EC POLYMER DEVICE}

PProDOT-Me $\mathrm{M}_{2}$ can be used as a cathodically coloring polymer and yields a dark blue color in its fully reduced form and a very transmissive light blue color in its oxidized form. In this case, a cathodically coloring polymer changes from a light color to a highly colored state upon charge neutralization (i.e., reduction) of the p-doped form. The $\pi-\pi^{*}$ transition is depleted at the expense of transitions outside the visible region. Therefore, the dominant wavelength of the color is the same throughout the doping process. ${ }^{11}$ The electrochromic process of the 


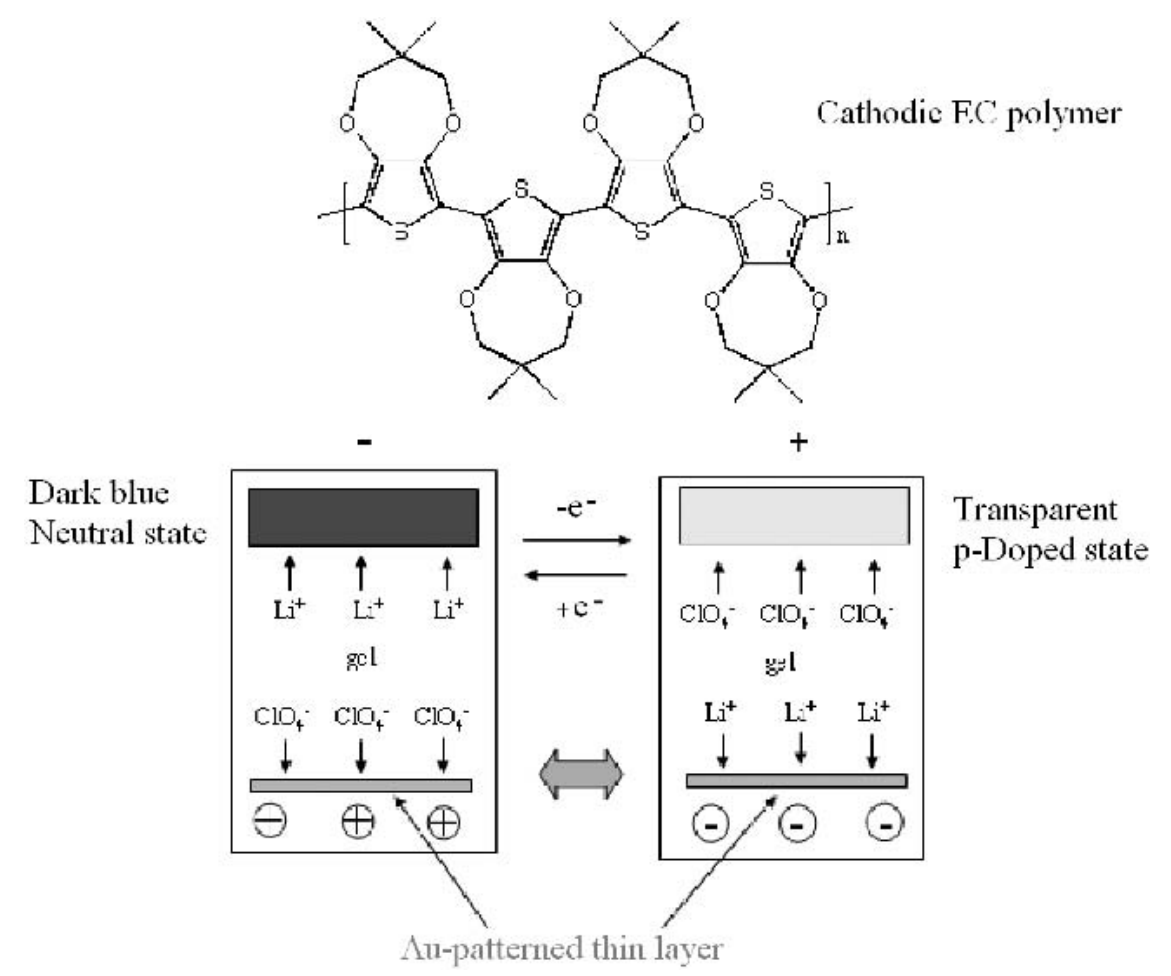

FIG. 4. Schematic diagram of the mechanism for color change of cathodic EC polymer, PProDOT-Me 2 .

EC window (ECW) fabricated with the Au-based counterelectrode can be summarized by the reaction scheme in Fig. 4, where Au is a gold layer playing a role in the formation of an electric double layer.

Inside the dashed-line frames, the EC polymer works with the Au-patterned thin layer as a pair. On the lefthand side, the EC polymer is charge neutralized, that is, it is reduced to a highly blue-colored state and the $\mathrm{Au}$ patterned thin layer works as an electric double layer as expressed by $\mathrm{Au} \oplus \ldots \mathrm{ClO}_{4}{ }^{-}$. On the right-hand side, the EC polymer is oxidized to the p-doped light color state. Here, the Au-patterned thin layer works as an electric double layer as expressed by $\mathrm{Au} \ominus \ldots \mathrm{Li}^{+}$. The gel electrolyte (gel in Fig. 4) is ionically conductive but electronically insulating. Hence, the $\mathrm{Li}^{+}$and $\mathrm{ClO}_{4}{ }^{-}$are mobile and easily move between the EC polymer side and the Au-patterned thin layer side under the polarity change of an applied potential.

The carbon patterned ITO glass-based counterelectrode also works by the same mechanism as the Au layer; it plays a part in the formation of an electric double layer.

In forming the electric double layer, no chemical reactions occur, causing no structural change in the Au or carbon-based materials. Also, the electric double layer can store both negative and positive charges. Therefore, the $\mathrm{Au}$ and carbon-based counterelectrodes will provide high electrochromic stability and are considered prime candidates for a counterelectrode of the ECW. ${ }^{8}$

\section{RESULTS AND DISCUSSION}

\section{A. Design of two-layer EC polymer device}

The conventional design of an ECW shown in Fig. 1 is a three-layer EC polymer window composed of dual polymers: a cathodic EC polymer and an anodic EC polymer. Here we propose a design of a two-layer structure EC polymer device, as shown in Fig. 5, composed of a cathodic EC polymer film and a gel electrolyte between two electrodes. ${ }^{2}$ The key components of the device are the EC polymer, the counterelectrode, and the gel electrolyte.

\section{B. Characterization and optimizing smart window components}

\section{Electropolymerization of four EC monomers}

Four EC monomers were successfully electropolymerized. Variable color electrochromic polymer films were

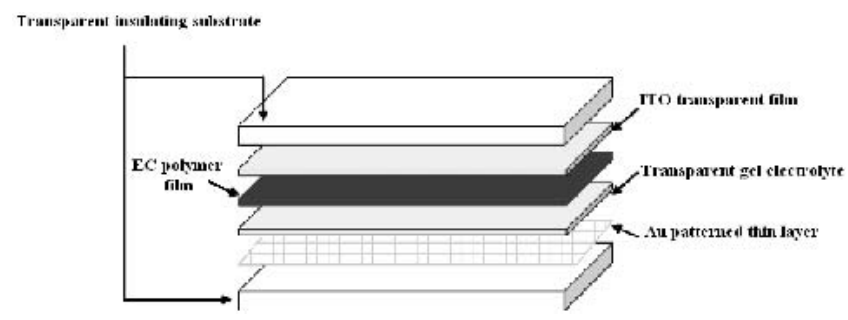

FIG. 5. Proposed ECW design with a gold-based glass counterelectrode. 
TABLE I. Colored and bleached states of two blue-color and two red-color EC polymers.

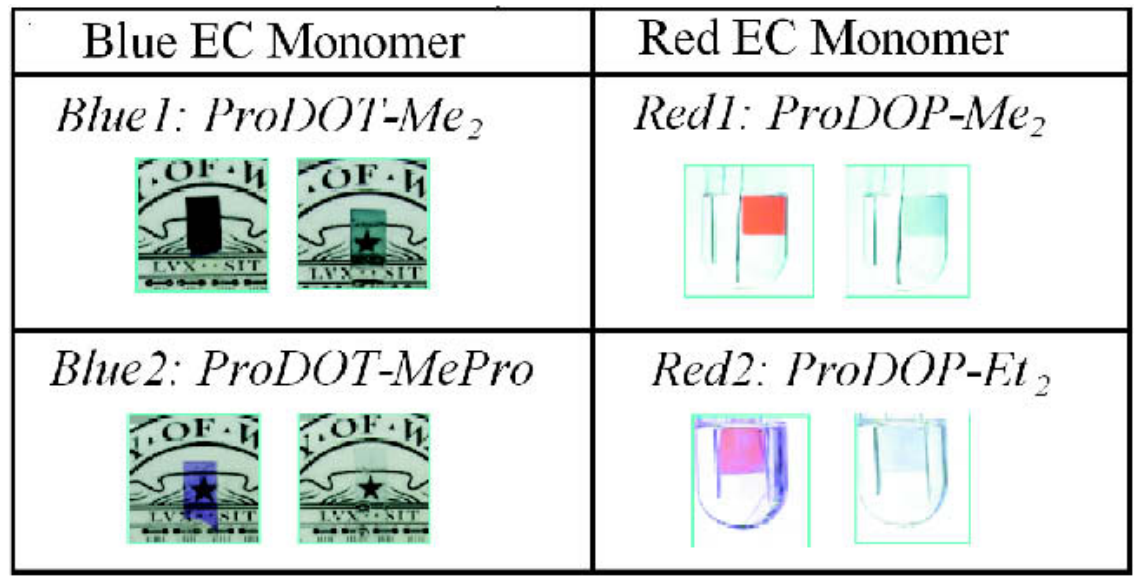

electrochemically deposited on ITO glass. Chronoamperometry and multiple cyclic voltammetry (CV) were used for the electropolymerization of the blue EC monomers and the red EC monomers, respectively. The parameters of the elctropolymerization were previously reported. ${ }^{1}$ Similar parameters were applied for blue 1 and 2 , red 1 and 2. Table I shows the resulting blue and red color EC films.

\section{Durability of the blue EC polymer}

Chronoamperometry and cyclic voltammetry were used to determine if the polymer degrades with cycling.

The blue $1 \mathrm{EC}$ monomer was electropolymerized on ITO glass $(7 \mathrm{~mm} \times 15 \mathrm{~mm})$ for $20 \mathrm{~s}$. This test was repeated for several film thicknesses, varied by increasing or decreasing the polymerization time and potential. The approximately 100 -nm film yielded the highest $\% \Delta \mathrm{T}$ (77\%), whereas the approximately $380-\mathrm{nm}$ film had a smaller $\% \Delta \mathrm{T}$ but could obtain an opaque transmittance close to $0 \%$, blocking out almost all transmitting light at the 580-nm wavelength.

Lifetime testing was performed on the polymer and ITO glass combination in an electrolyte solution. The EC polymer successfully switched between the fully oxidized and reduced states for 50,000 cycles in a $0.1 \mathrm{M} \mathrm{PC}$ and $\mathrm{LiClO}_{4}$ electrolyte solution that was sufficiently dried, as noted in Sec. II. B. Figure 6 shows the change in CV curve initially and after 50,000 cycles.

\section{Optimization and durability of gel electrolyte}

Lithium (Li) is commonly used in switching devices due to its small size and ease in reducing and oxidizing electronic polymers. Another salt, TBAP, was also used for comparison. Overall, the salt must have a high degree of dissociation and the anion must have a high level of charge delocalization so that the ion-pairing is minimized. ${ }^{13}$ Seven kinds of gel were prepared as described elsewhere. ${ }^{17}$ The ionic conductivity measurements were

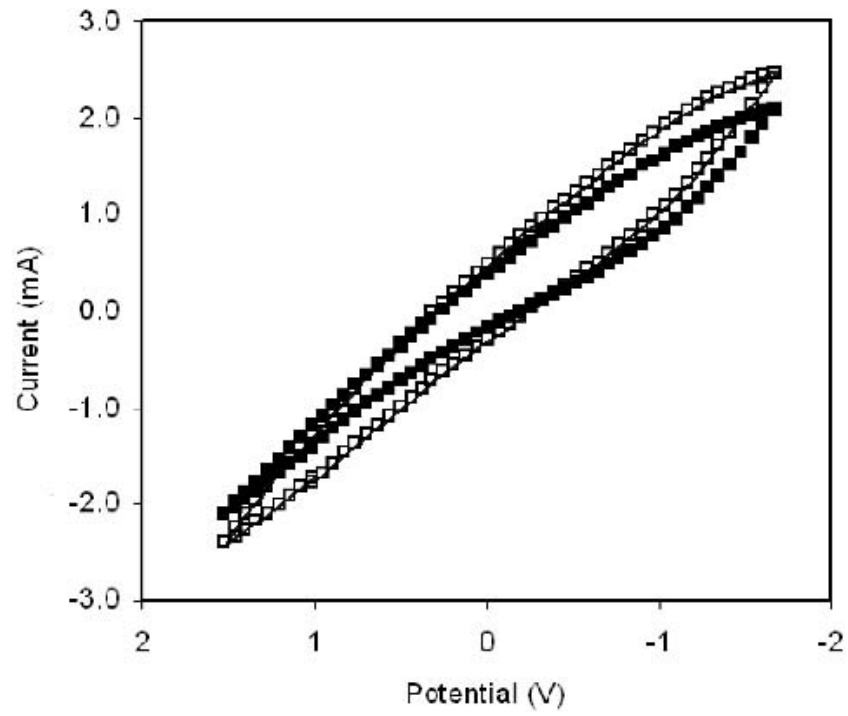

FIG. 6. Initial CV curve ( $\square$ ) and CV curve after 50,000 (ם) cycles for an approximately $100-\mathrm{nm}$ film.

recorded at ambient temperature for 90 days, as exhibited in Fig. 7. Of the seven different candidates, GBL/PC/ $\mathrm{LiClO}_{4} / \mathrm{PMMA}$ and $\mathrm{EC}+\mathrm{PC} / \mathrm{LiClO}_{4} / \mathrm{PMMA}$ were found to be the most promising ones for the smart window device due to their high ionic conductivities, transmittances, and stability.

The lifetime of the EC polymer film was successfully tested in both an electrolyte solution, as shown in Fig. 8, and also in a gel electrolyte for 50,000 cycles. A close examination of the $\mathrm{CV}$ curves reveals that most of the film degradation occurred within the first 5000 cycles. After 5000 cycles, the rate of degradation significantly decreased. From 25,000 to 50,000 cycles, minimal degradation is observed. One possible explanation of the degradation is due to residual moisture in the electrochemical cell and on the EC film, which can be concluded by the presence of bubbles that formed around the counterelectrode. 


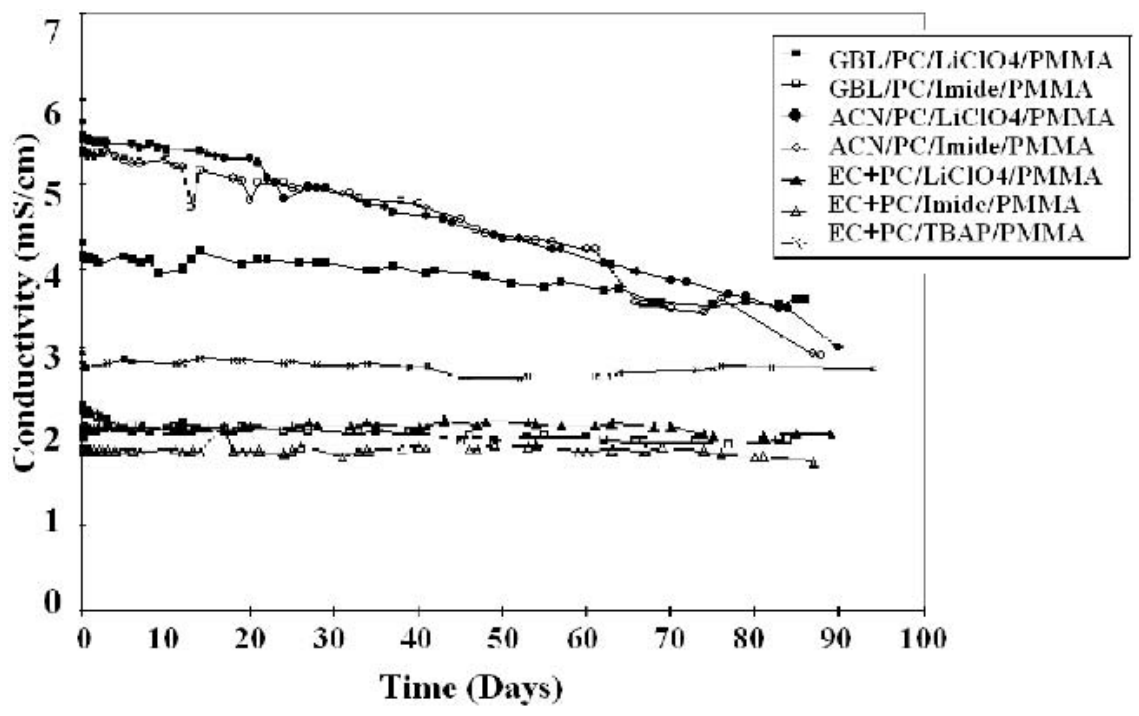

FIG. 7. Conductivity of gel electrolyte candidates over time.

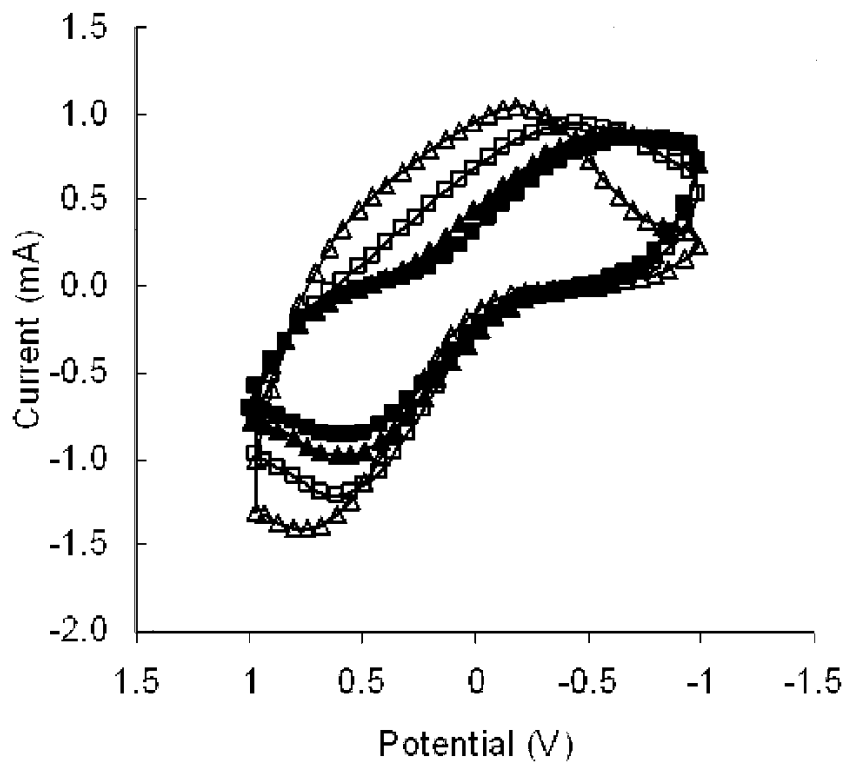

FIG. 8. CV curve for EC polymer on ITO glass initially $(\triangle)$, after 10,000 cycles $(\square), 25,000$ cycles $(\boldsymbol{\Delta})$, and 50,000 cycles $(\boldsymbol{\square})$.

\section{Optimization and durability of counterelectrode}

The counterelectrode material should be chemically stable, providing high electrical conductivity and ease of processing into a patterned substrate. Thus, Au and carbon were selected as the electrically conductive materials for making the counterelectrode. Because electric conductivity of $\mathrm{Au}$ is much higher than carbon, Au was directly deposited on the glass without ITO, while graphite was patterned on glasses with ITO as described in Sec. II. C. The wider width of conductive material line and the smaller open area of the grid pattern are expected to provide higher conductivity, thus enhancing the color

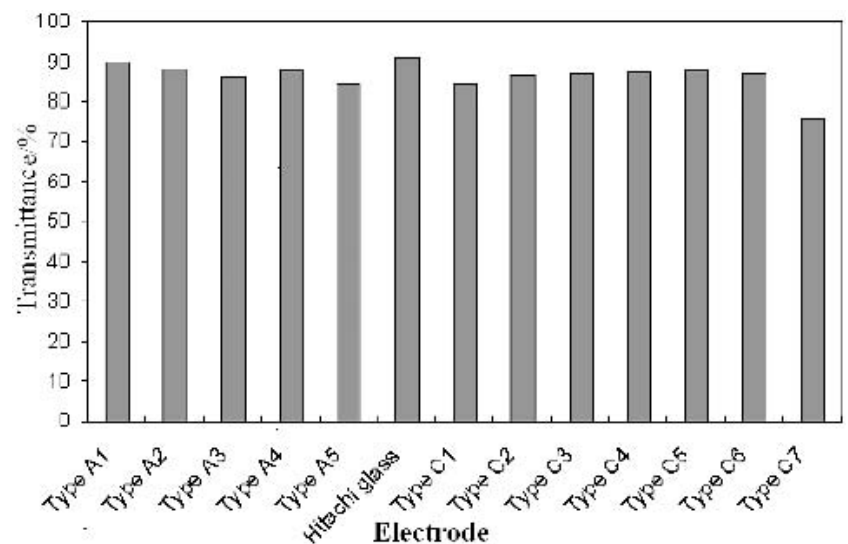

FIG. 9. Transmittance data of counterelectrodes.

change speed of EC polymer, but at the cost of decreasing light transmittance of the counterelectrode. Therefore, the use of a highly conductive material and optimum pattern geometry of the electrode are key design parameters. As a result, the graphite patterned with ITO glass substrate was used as the carbon-based counterelectrode for subsequent experiments. ${ }^{2}$

Transmittances of five Au-based (A1-A5) and seven carbon-based (C1-C7) counterelectrodes of different patterns and geometry were characterized. The percent transmittance $(\% \mathrm{~T})$ of the counterelectrodes was measured and compared to the transmittance of optical glass (Hitachi glass provided by HPM). The results are shown in Fig. 9. Both the Au-based and carbon-based ITO counterelectrodes exhibit high transmittance, as shown by the high \% compared to the Hitachi glass. The only exception was that of the carbon-based ITO sample with a $6-\mu \mathrm{m}$ line width and $0.1-\mathrm{mm}$ window size. The low transmittance of $75 \%$ is a result of the large amount of carbon present on the counterelectrode. 


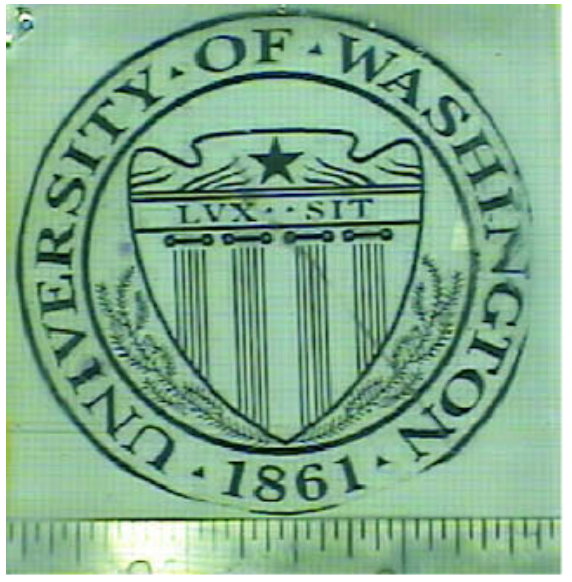

(a)

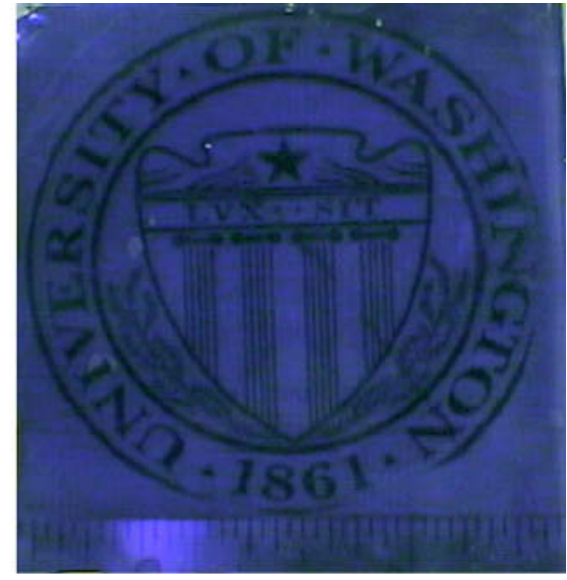

(b)

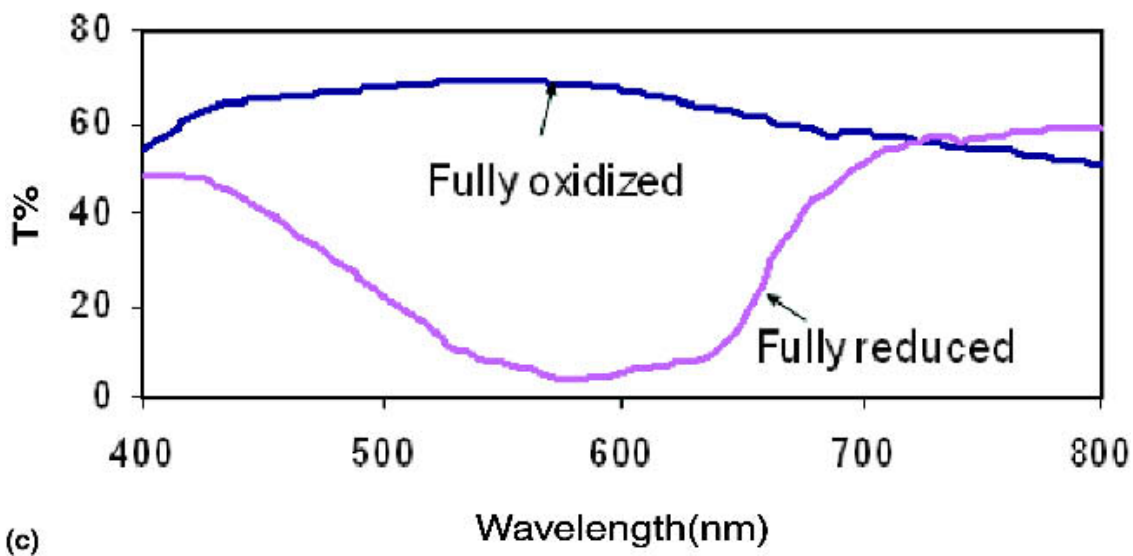

FIG. 10. 3-inch $\times$ 3-inch assembled device: (a) fully oxidized state and (b) fully reduced state; and (c) transmittance of the device at visible region in two states.

The stability of the counterelectrode over a lifetime of 10,000 cycles was tested using CV. Because the change in the CV curve after 10,000 was minimal for both cases, it was concluded that the counterelectrode exhibit little degradation.

\section{Assembly and color change of device}

After the three components were well characterized, a device was assembled. Figure 10 shows transparent and colored stages of this device. The device has a transmittance contrast ratio of over $60 \%$ in the wavelength of $580 \mathrm{~nm}$.

It was observed that a higher electric field and longer time are needed to switch the device between the opaque and transparent states $( \pm 2.8 \mathrm{~V}$ for $4 \mathrm{~s})$ compared to that required to switch the EC polymer film in an electrolyte solution $( \pm 1.0 \mathrm{~V}$ for $1 \mathrm{~s})$. An alternative device was made to further investigate the reason for the higher potentials. As shown in Fig. 11, an additional Ag foil as a reference electrode was placed in contact with the gel electrolyte but separated from the counterelectrode and EC film polymerized on ITO glass by parafilm. This design imitates

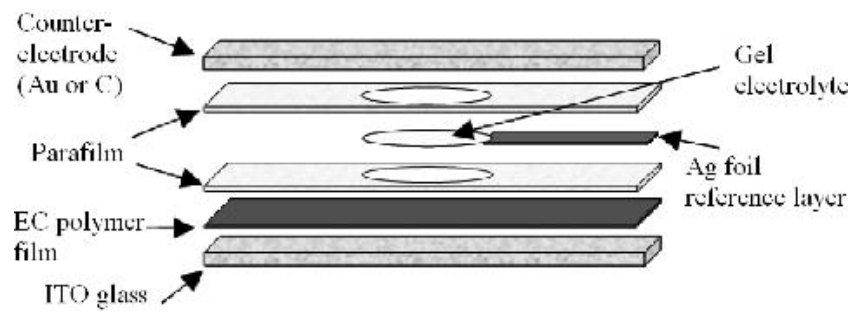

FIG. 11. A cross-sectional view of the redesigned device.

a solution type cell (three electrodes) that was used during testing before assembly as a solid device. A $1.0 \mathrm{~V}$ E-field was found to result in good repeatability $(75,000$ cycles) without damaging the EC film within the solution type cell. The final device uses a gel electrolyte instead of solution electrolyte because of its semi-solid state. The ionic conductivity of the gel electrolyte is lower than that of the solution electrolyte which was used in solution type cell. The addition of an $\mathrm{Ag}$ foil reference in the solid device provides quick feedback to the control system to enhance control of the E-field and, consequently, device performance. 
The resulting device switched at lower potentials and achieved the same transmittance change $\% \triangle \mathrm{T}(60 \%)$ (Fig. 12) in $1 \mathrm{~s}$ as the device assembled without the $\mathrm{Ag}$ foil. It is also noted that operating the device with a reference electrode allows for applied voltage to remain constant but can also prevent damage to the EC film, which can occur sometimes when a potential greater than $3 \mathrm{~V}$ is applied in the EC film side during electrochemical reaction.

Two 3-inch $\times 3$-inch devices were also prepared: with and without the reference layer for comparison. Both devices achieved a transmittance change of $\% \Delta \mathrm{T}$ over $60 \%$; the applied potentials and step times differed significantly. By adding the additional reference layer, the potential of the 3-inch $\times 3$-inch device was well controlled to $1.0 \mathrm{~V}$ between reference and working electrode, and the coloration time dropped from $8 \mathrm{~s}$ to $1 \mathrm{~s}$.

\section{Temperature dependence}

Temperature dependence of the color change performance of ECs is also an important factor in designing EC devices for use in various temperature environments. This has been reported elsewhere. ${ }^{1,2}$ It is stable and wide, $-40 \sim 100{ }^{\circ} \mathrm{C}$.

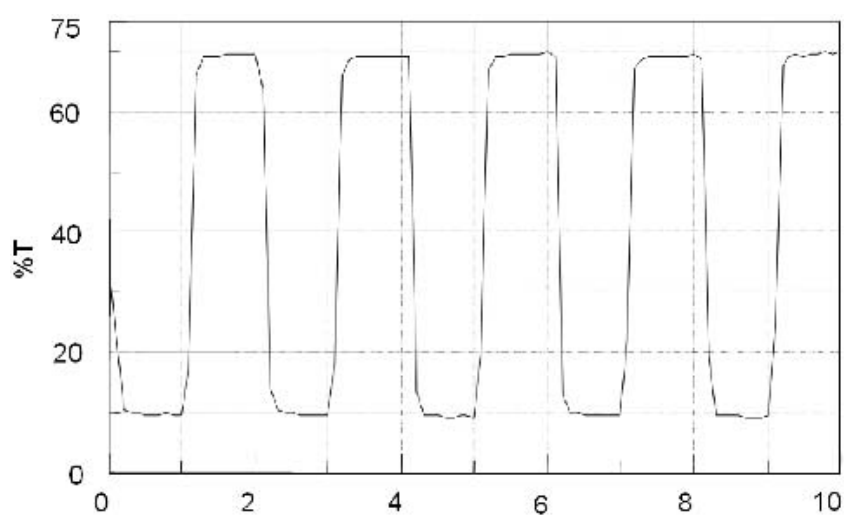

(a) Time [Sec]

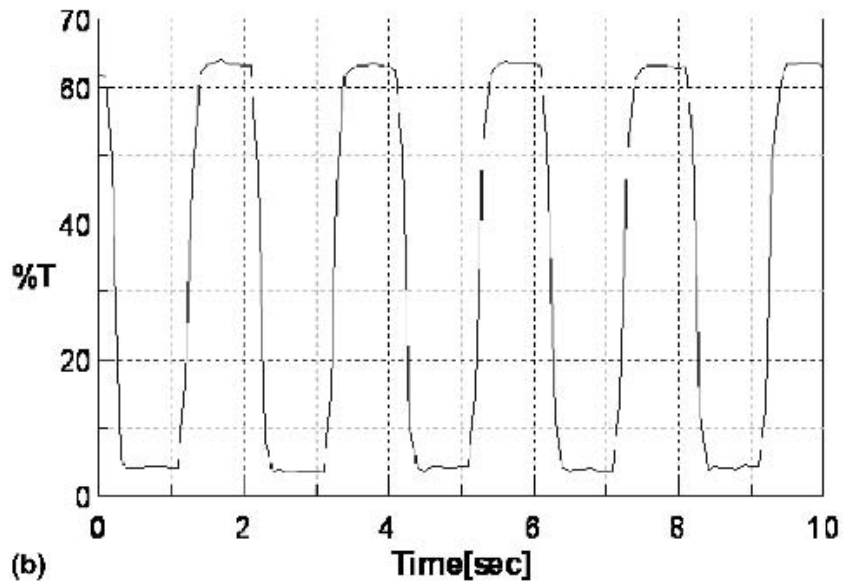

FIG. 12. The transmittance data for the 1 -inch $\times 1$-inch (a) carbon-based and (b) Au-based devices under switching at a fixed wavelength $580 \mathrm{~nm}$.

\section{CONCLUSIONS}

An EC polymer device that consists of a laminated two-layer structure between two electrodes was prepared. The new design, which consists of an ITO glass electrode, a cathodic EC polymer film, a solid electrolyte, and an Au-based (or carbon-based) counterelectrode, is simpler than the current design of EC device that consists of an additional component (i.e., anodic EC polymer).

One brand-new blue (ProDOT-MePro) and one brandnew red (ProDOP-Et ${ }_{2}$ ) color changeable EC monomers each was successfully synthesized and their EC polymer films were formed by electropolymerization. High contrast ratio $\% \triangle \mathrm{T}$ of 3-inch $\times 3$-inch blue color rapidly switchable device was prepared for the first time.

The speed of switching the proposed EC window between transparent and colored is rapid, less than $1.0 \mathrm{~s}$ for 1-inch $\times 1$-inch device. Carbon-based counterelectrode with ITO can achieve faster response time than Au-based counterelectrode. The blue 1-color EC polymer showed high repeatability (50,000 cycles).

In addition, the wide temperature ranges under which the switching is stable are presented for the first time. This allows for the Au-based cell and carbon-based cell to be useful for extreme environment applications.

The above EC polymer devices provide a memory effect, good perceived contrast, and low switching voltage. The electrochromic devices are of special interest for use in dialed-tint windows, large-area displays, and antiglare car rear-view mirrors.

\section{ACKNOWLEDGMENTS}

This work was conducted under contract from a corporation at the University of Washington, where discussions with Mr. Art Weidner and Dr. Thomas A. Woodrow are greatly appreciated. The authors are thankful to Mr. H. Chiyoda and Mr. S. Deyama of Hitachi Powder Metals Co. Ltd. for their assistance in providing the carbon-based counterelectrodes.

\section{REFERENCES}

1. C. Xu, L. Liu, S. Legenski, M. Le Guilly, M. Taya, and A. Weidner: Enhanced smart window based on electrochromic (EC) polymers. Smart Structures and Materials 2003. Proceeding of SPIE. 5051, 404 (2003).

2. C. Xu, H. Tamagawa, M. Uchida, and M. Taya: Enhanced contrast ratios and rapid switching color changeable devices based on poly(3,4-propylenedioxythiophene) derivative and counterelectrode. Smart Structures and Materials 2002. Proceeding of SPIE. 4695, 442 (2002).

3. C.M. Lampert: Large-area smart glass and integrated photovoltaics. Solar Energy Mater. 76, 489 (2003).

4. D. Lundstrom and J. Yilbar: Liquid crystal materials: Experimental material physics. Available at http://www.kth.se/fakulteter/tfy/ $\mathrm{kmf} / \mathrm{lcd} / \mathrm{lcd} 1 . \mathrm{htm}$. 
5. B.C. Thompson, P. Schottland, K. Zong, and J.R. Reynolds: In situ colorimetric analysis of electrochromic polymers and devices. Chem. Mater. 12, 1563 (2000).

6. S.A. Sapp, G.A. Sotzing, and J.R. Reynolds: High contrast ratio and fast-swiching dual polymer electrochromic devices. Chem. Mater. 10, 2101 (1998).

7. D.M. Welsh, A. Kumar, E.W. Meijer, and J.R. Reynolds: Enhanced contrast ratios and rapid switching in electrochromics based on poly(3,4-propylenedioxythiophene) derivatives. Adv. Mater. 11, 1379 (1999).

8. Y. Nishikitani, T. Asano, S. Uchida, and T. Kuba: Thermal and optical behavior of electrochromic windows fabricated with carbon-based counterelectrode. Electrochim. Acta. 44, 3211 (1999).

9. G.A. Sotzing, J.L. Reddinger, A.R. Katritzky, J. Soloducho, R. Musgrave, J.R. Reynolds, and P.J. Steel: Multiply colored electrochromic carbazole-based polymers. Chem. Mater. 9, 1578 (1997).

10. M. Lapkowski and A. Pron: Electrochemical oxidation of poly (3,4-ethylenedioxythiophene) - "in situ" conductivity and spectroscopic investigations. Synth. Met. 110, 79 (2000).
11. I. Schwendeman, J.H. Hwang, D.M. Welsh, D.B. Tanner, and J.R. Reynolds: Combined visible and infrared electrochromism using dual polymer devices. Adv. Mater. 13, 634 (2001).

12. A. Merz, R. Schropp, and E. Dotterl: 3,4-Dialkoxypyrroles and 2,3,7,8,12,13,17,18-octaalkoxyporphyrins. Synthesis 7, 795 (1995).

13. S.A. Agnihotry and S.S. Pradeep: Sekhon, PMMA based gel electrolyte for EC smart windows. Electrochim. Acta. 44, 3121 (1999).

14. L. Su, J. Fang, Z. Xiao, and Z. Lu: An all-solid-state Electrochromic display device of prussian blue and $\mathrm{WO}_{3}$ particulate film with a PMMA gel electrolyte. Thin Solid Films 306, 133 (1997).

15. L. Su, J. Fang, Z. Xiao, and Z. Lu: All solid-state elecrtochromic window of electrodeposited $\mathrm{WO}_{3}$ and Prussian blue film with PVC gel electrolyte. Thin Solid Films 320, 285 (1998).

16. S.S. Sekhon and S.A. Deepa: Agnihotry, solvent effect on gel electrolytes containing lithium salts. Solid State Ionics 136, 1189 (2000).

17. S. Legenski, C. Xu, L. Liu, M. Le Guilly, and M. Taya, Gel electrolyte candidates for electrochromic devices (ECDs), Smart Structures and Material, Proc. of SPIE. 5385 (2004, in press). 